\title{
A spiking temporal-difference learning model based on dopamine-modulated plasticity Wiebke Potjansu ${ }^{* 1,2}$, Abigail Morrison ${ }^{1}$ and Markus Diesmann ${ }^{1,3,4}$
}

\author{
Address: ${ }^{1}$ Theoretical Neuroscience Group, RIKEN Brain Science Institute, Wako City, Saitama, Japan, ${ }^{2}$ Institute of Neurosciences and Medicine, \\ Research Center Jülich, Jülich, Germany, ${ }^{3}$ Brain and Neural Systems Team, RIKEN Computational Science Research Program, Wako City, Saitama, \\ Japan and ${ }^{4}$ Bernstein Center for Computational Neuroscience, Albert-Ludwigs-University, Freiburg, Germany \\ Email: Abigail Morrison - abigail@brain.riken.jp \\ * Corresponding author
}

from Eighteenth Annual Computational Neuroscience Meeting: CNS*2009

Berlin, Germany. 18-23 July 2009

Published: 13 July 2009

BMC Neuroscience 2009, I0(SuppI I):PI40 doi:I0.II86/I47I-2202-I0-SI-PI 40

This abstract is available from: http://www.biomedcentral.com/I47I-2202// 0/SI/PI40

(c) 2009 Potjansu et al; licensee BioMed Central Ltd.

Making predictions about future rewards and adapting the behavior accordingly is crucial for any higher organism. One theory specialized for prediction problems is temporal-difference (TD) learning. Experimental findings suggest that TD learning is implemented by the mammalian brain. In particular, the resemblance of dopaminergic activity to the TD error signal [1] and the modulation of corticostriatal plasticity by dopamine [2] lend support to this hypothesis. We recently proposed the first spiking neural network model to implement actor-critic TD learning [3], enabling it to solve a complex task with sparse rewards. However, this model calculates an approximation of the TD error signal in each synapse, rather than utilizing a neuromodulatory system.

Here, we propose a spiking neural network model which dynamically generates a dopamine signal based on the actor-critic architecture proposed by Houk [4]. This signal modulates as a third factor the plasticity of the synapses encoding value function and policy. The proposed model simultaneously accounts for multiple experimental results, such as the generation of a TD-like dopaminergic signal with realistic firing rates in conditioning protocols [1], and the role of presynaptic activity, postsynaptic activity and dopamine in the plasticity of corticostriatal synapses [5]. The excellent agreement between the predictions of our synaptic plasticity rules and the experimental findings is particularly noteworthy, as the update rules were postulated employing a purely top-down approach.

We performed simulations in NEST [6] to test the learning behavior of the model in a two dimensional grid-world task with a single rewarded state. The network learns to evaluate the states with respect to its reward proximity and adapt its policy accordingly. The learning speed and equilibrium performance are comparable to those of a discrete time algorithmic TD learning implementation.

The proposed model paves the way for investigations of the role of the dynamics of the dopaminergic system in reward-based learning. For example, we can use lesion studies to analyze the effects of dopamine treatment in Parkinson's patients. Finally, the experimentally constrained model can be used as the centerpiece of closedloop functional models.

\section{Acknowledgements}

Partially funded by EU Grant 15879 (FACETS), BMBF Grant 0 I GQ0420 to BCCN Freiburg, Next-Generation Supercomputer Project of MEXT, Japan, and the Helmholtz Alliance on Systems Biology.

\section{References}

I. Schultz W, Dayan P, Montague PR: A neural substrate of prediction and reward. Science 1997, 275:1593-1599.

2. Reynolds JN, Hyland BI, Wickens JR: A cellular mechanism of reward-related learning. Nature 200I, 4I3:67-70. 
3. Potjans W, Morrison A, Diesmann M: A spiking neural network model of an actor-critic learning agent. Neural Computation 2009, 21:301-339.

4. Houk JC, Adams JL, Barto AG: A model of how the basal ganglia generate and use neural signals that predict reinforcement MIT Press, Cambridge, MA; 1995.

5. Reynolds JN, Hyland BI, Wickens JR: Dopamine-dependent plasticity of corticostriatal synapses. Neural Networks 2002, | 5:507-52|.

6. Gewaltig M-O, Diesmann M: NEST (neural simulation tool) Scholarpedia 2007, 2:1430.

Publish with Bio Med Central and every scientist can read your work free of charge

"BioMed Central will be the most significant development for disseminating the results of biomedical research in our lifetime. " Sir Paul Nurse, Cancer Research UK

Your research papers will be:

- available free of charge to the entire biomedical community

- peer reviewed and published immediately upon acceptance

- cited in PubMed and archived on PubMed Central

- yours - you keep the copyright

Submit your manuscript here:

http://www.biomedcentral.com/info/publishing_adv.asp 